EPJ Web of Conferences 61, 03002 (2013)

DOI: $10.1051 /$ epjconf/ 20136103002

(C) Owned by the authors, published by EDP Sciences, 2013

\title{
Search for new stellar sources of gamma-rays
}

\author{
Josep Martí ${ }^{1,3, a}$, Estrella Sánchez-Ayaso ${ }^{3, b}$, Pedro L. Luque-Escamilla ${ }^{2,3, c}$, and Juan R. Sánchez-Sutil ${ }^{3, d}$ \\ ${ }^{1}$ Universidad de Jaén, Escuela Politécnica Superior de Jaén, Departamento de Física, Campus Las Lagunillas s/n, A3, 23071 \\ Jaén, Spain \\ ${ }^{2}$ Universidad de Jaén, Escuela Politécnica Superior de Jaén, Departamento de Ingeniería Mecánica y Minera, Campus Las \\ Lagunillas s/n, A3, 23071 Jaén, Spain \\ ${ }^{3}$ Grupo de Investigación FQM-322, Universidad de Jaén, Campus Las Lagunillas s/n, Edif. A3, 23071 Jaén, Spain
}

\begin{abstract}
We review and report about the present status of our search for gamma-ray binaries, microquasars, and new kinds of gamma-ray source associated with star forming regions in the Galaxy. The search is being carried out using cross-identification techniques applied to public databases and archives. A few promising candidates have been so far identified. These include the emission line star VES 737 and the central cluster of the Monoceros R2 star forming region. The observational data supporting the proposed associations is shortly presented and discussed.
\end{abstract}

\section{Introduction}

The zoo of Galactic gamma-ray sources has expanded in the recent years thanks to the new generation of highenergy observatories. Instruments such as the Fermi Gamma Ray Space Telescope ${ }^{1}$, or the ground-based atmospheric imaging Cherenkov telescopes MAGIC ${ }^{2}$, H.E.S.S. ${ }^{3}$, and VERITAS ${ }^{4}$, among others, have been key to unveil new kinds of energetic sources. Outstanding among them there are the so called 'gamma-ray binaries' (see e.g. [1] for a review). In parallel, other stellar gammaray sources have been predicted to exist based on theoretical work, although their firm detection remains yet to be accomplished. These include T-Tauri stars [2], bipolar outflows from massive protostars [3] as well as bowshocks from runaway stars [4].

Here we provide a brief account of our search efforts aimed to identify particular examples of these previously unknown Galactic gamma-ray emitters. Our approach is mainly based on exploring multi-wavelength databases and archives, with eventual follow up observations when some interesting candidate emerges. In particular, we have been trying to find out additional members of the gamma-ray binary group, whose currently known population barely amounts to a half dozen cases. Nearby star forming regions have also subject to a deep scrutiny to maximize the chances of detecting any of the gamma-ray

\footnotetext{
ae-mail: jmarti@ujaen.es

be-mail: eayaso@ujaen.es

ce-mail: peter@ujaen.es

de-mail: jrssutil@ujaen.es

${ }^{1}$ http: //fermi.gsfc.nasa.gov

${ }^{2}$ https://wwwmagic.mpp.mpg.de

${ }^{3}$ http: //www.mpi-hd.mpg.de/hfm/HESS/

${ }^{4}$ http://veritas.sao.arizona.edu
}

sources quoted above associated with young or massive stars. This work is complementary of other similar efforts in this direction [5].

\section{Search for new gamma-ray binary systems}

Currently known gamma-ray binaries, such as LS +61 303, HESS J0632+057, and the microquasar Cygnus X-1, correspond to different entries in the catalogue of luminous stars in the Northern Milky Way [6]. The proposed optical counterpart to HESS J0632+057 is also noted as MWC 148 in the catalogue of early-type stars whose spectra have shown emission lines [7]. The same occurs with AGL J2241+4454, whose bright optical counterpart is believed to coincide with the star MWC 656. The well-known gamma-ray binary LS 5039, another entry in the [6] catalogues, has a non-thermal radio counterpart first noticed by some of the authors after inspection of archival survey data [8]. In this context, we speculate if similar systems could be waiting for discovery hidden in these or other similar old catalogues.

In an attempt to contribute with new findings, we have undertaken a systematic search using different optical, radio, X-ray, and gamma-ray catalogues in the public domain ${ }^{5}$. Our search approach follows the path of previous cross-identification techniques developed by our group (see e.g. [9]). The ideal gamma-ray binary/microquasar candidate would be an early-type star found to be reliably consistent in position with a non-thermal radio source, an $\mathrm{X}$-ray source, and eventually a gamma-ray source unassociated at lower energies. Correlated variability be-

\footnotetext{
${ }^{5}$ http://cdsarc.u-strasbg.fr/cats/Cats.htx
} 
Table 1. Preliminary results of cross-Id search

\begin{tabular}{|c|c|c|}
\hline Gamma-ray source & $\begin{array}{l}\text { Candidate } \\
\gamma \text {-ray binary }\end{array}$ & Remarks \\
\hline $\begin{array}{l}\text { 1FGL J2056.7+4938/ } \\
\text { 2FGL J2056.7+4939 }\end{array}$ & LS III +49 13 & $\begin{array}{l}\text { Star already proposed as possible microquasar by [12]; } \\
\text { [13] suggest } 47 \% \text { chance of coincidence with ROSAT } \\
\text { source; Fermi-LAT source could be an AGN. }\end{array}$ \\
\hline $\begin{array}{l}\text { 1FGL J0608.1-0630c/ } \\
\text { 2FGL J0607.5-0618c }\end{array}$ & LS IV -06 1 & $\begin{array}{l}\text { Unassociated Fermi-LAT source; star in nebula; } \\
\text { Possible NVSS radio counterpart; no X-ray detection }\end{array}$ \\
\hline $\begin{array}{l}\text { 1FGL J1823.2-1336c/ } \\
\text { 2FGL J1823.1-1338c }\end{array}$ & LS 4995 & $\begin{array}{l}\text { Unassociated Fermi-LAT source; candidate star is an } \\
\text { early-type supergiant; no X-ray nor radio detection in archives. }\end{array}$ \\
\hline $\begin{array}{l}\text { 1FGL J0220.0+6257/ } \\
\text { 2FGL J0221.4+6257c }\end{array}$ & VES 737 & $\begin{array}{l}\text { Fermi-LAT source could be associated with supernova } \\
\text { remnant, but this is not clear. VES } 737 \text { is an early-type } \\
\text { emission line star with a possible X-ray detection by EINSTEIN. }\end{array}$ \\
\hline
\end{tabular}

tween the different wavelength domains would add further strength to the identification. Unfortunately, all these requirements are extremely difficult to be simultaneously fullfiled in practice.

The Fermi Large Area Telescope (LAT) catalogues have been used in this search as a starting point, specially the 2FGL catalogue based on the first two years of satellite data [10]. Unassociated Fermi-LAT sources were extensively cross-identified with all of the peculiar star catalogues mentioned above. As a result, and with all caution, we report a few sources that we consider as candidate gamma-ray binaries worth to be further investigated. They are listed in Table 1, where first column indicates the gamma-ray source name(s), second column gives the name of the star tentatively associated with it, and third column lists any relevant remark.

Among the four stars in Table 1, the case of the Btype emission line star VES 737 [11] is our highest priority finding because of the additional coincidence with the EINSTEIN X-ray source 2E $0216.9+6248$. This star is located a J2000.0 coordinates $\alpha=02: 20: 46.853$ and $\delta=+63: 01: 17.42$. With a visual magnitude of $V=11.86$, it is the brightest optical star located close to the edge but inside the EINSTEIN error circle (see Fig. 1). The VES737 position is unfortunately not well covered by modern X-ray satellites (XMM-Newton and Chandra).

Follow-up observations to confirm the proposed stars tentatively associated with gamma-ray sources need to be carried out. In particular, we aim at deep radio searches for non-thermal emission and period analysis of public FermiLAT light curves to look for orbital period signatures. So far a short exploratory radio observation of the VES 737 field has been carried out (on 2011 June 17) with the Jansky Expanded VLA Array (EVLA) to search for a radio counterpart, although with negative results (see Fig. 2). The instrumental setup used the L-band $(20 \mathrm{~cm})$ receivers with two spectral windows of $128 \mathrm{MHz}$ bandwidth divided into 64 channels each, and the most extended A configuration of the array that provides arc-second angular resolution. The rms noise achieved using natural weight was $27.6 \mu \mathrm{Jy}$, which implies a $3 \sigma$ upper limit of $0.083 \mathrm{mJy}$ at the VES 737 position. The closest radio source detected was located at J2000.0 coordinates $\alpha=02: 20: 51.914$ and $\delta=+63: 01: 10.29$, with a $20 \mathrm{~cm}$ flux density of $1.9 \mathrm{mJy}$.

\section{Search for new kinds of gamma-ray sources in star forming regions}

The nearby ( $\leq 1 \mathrm{kpc}$ ) star forming regions Monoceros $\mathrm{R} 2$ and $\rho$ Ophiuchi appear to be in the direction of the Fermi-LAT sources 2FGL J0607.5-0618c and 1FGL J1625.8-2429c, respectively. The possibility of a true association beyond apparent coincidence has been seriously proposed based on physical arguments [2, 15], although a firm proof of it is not yet available. Nevertheless, one has still to be cautious even with the reality of the gammaray sources themselves. This is because its proximity to the Galactic plane renders extremely difficult to model the gamma-ray background, and hence the possibility of the Fermi-LAT source being spurious cannot be ruled out. Indeed, this is the reason for the 'c' flag in the source names.

The following subsections are devoted to summarize the most relevant information about these two fields.

\subsection{The Monoceros R2 case}

The in depth study of 2FGL J0607.5-0618c by [15] started by confirming the reality of the gamma-ray source by analyzing the whole Fermi-LAT data set available. As a result, the source detection was improved at the $\sim 12 \sigma$ level as compared to the less significant detection in the 2FGL catalogue.

The Monoceros R2 central cluster region, rich in young stellar objects (YSOs) including T-Tauri stars and massive protostars, is the most relevant object inside the Fermi-LAT confidence ellipse (see Fig. 3). If the gammaray emission is not due to a collective effect of YSOs in the region, then one would be forced to assume an anomalous enhancement of the local cosmic ray energy by a factor of $\sim 7$ [15]. No alternative background/foreground counterpart unrelated to Monoceros R2 appears obvious in multiwavelength data of the region.

\subsection{The $\rho$-Ophiuchi case}

This very nearby star forming region appeared a few years ago as a promising candidate for gamma-ray emission produced by an ensemble of T-Tauri stars. The energetic photons, up to $\mathrm{TeV}$ energies, could eventually be produced 


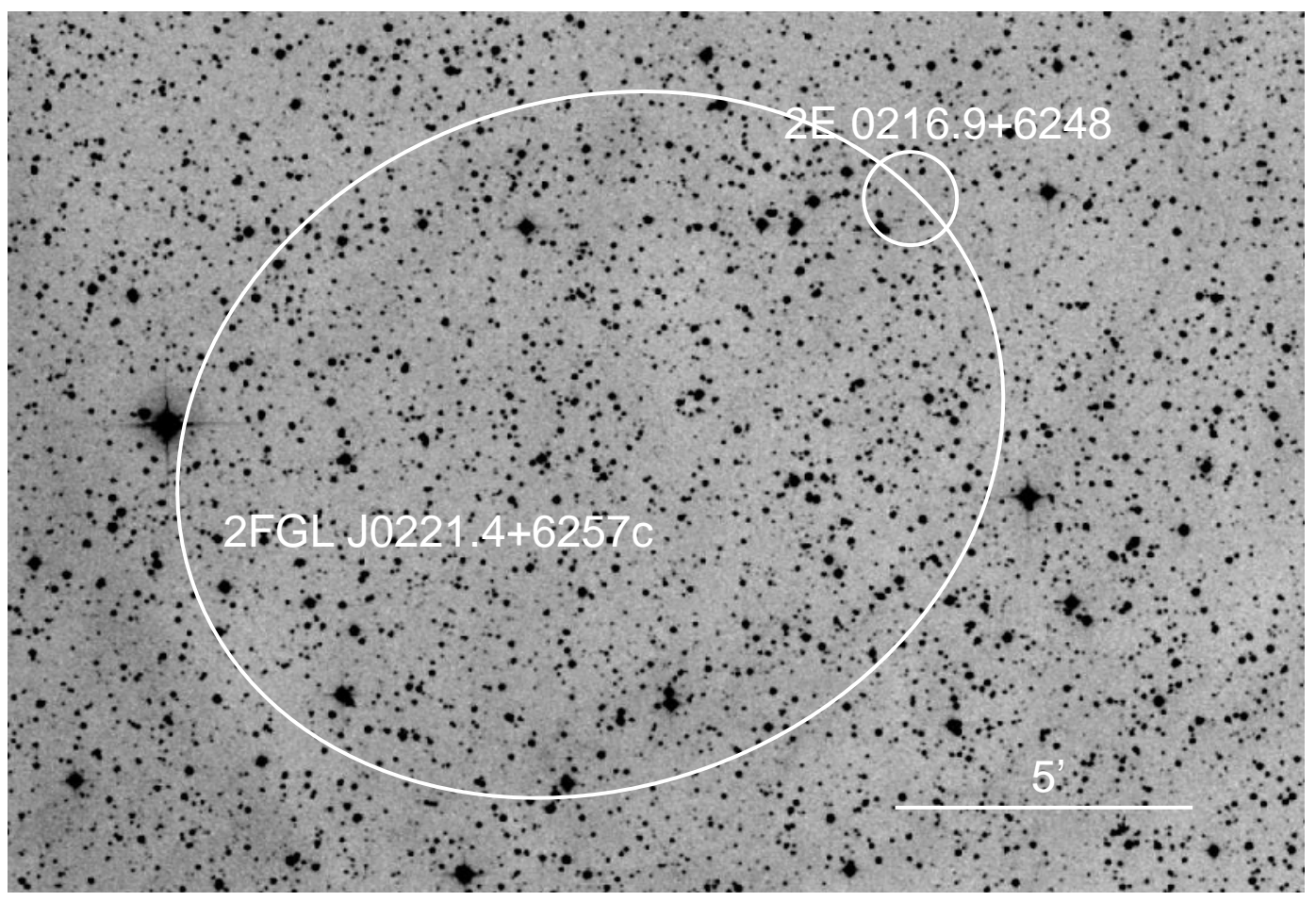

Figure 1. Optical image from the Second Palomar Observatory Sky Survey (POSS-II, red plate) showing the error ellipses for the Fermi-LAT and EINSTEIN X-ray sources 2FGL J0221.4+6257c and 2E 0216.9+6248, respectively. The emission line star VES 737, the brightest optical object inside the EINSTEIN smaller error circle, is consistent with both of them.

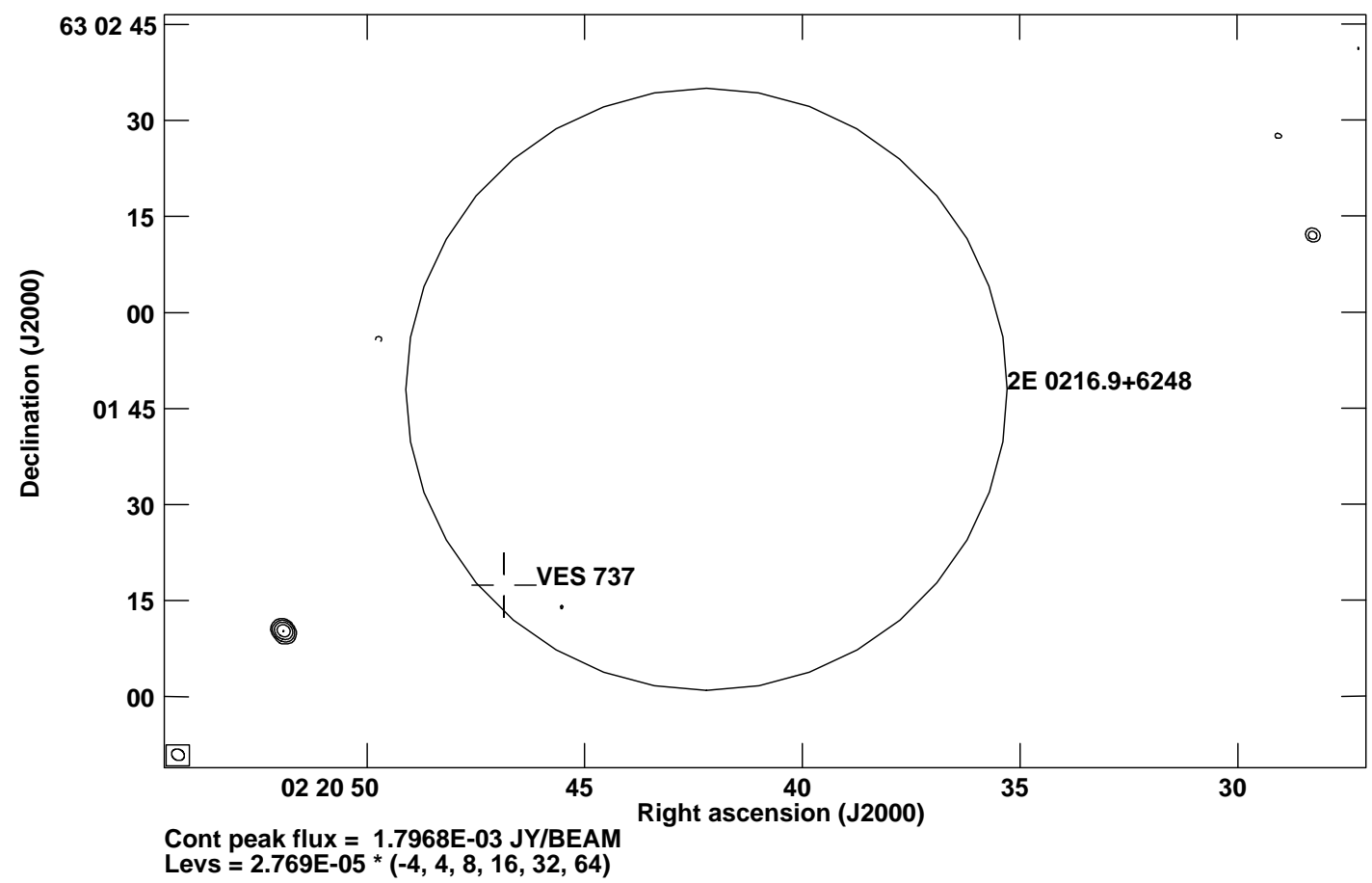

Figure 2. Contour radio map of the VES 737 position obtained with the Jansky EVLA at the $20 \mathrm{~cm}$ wavelength. The circle represents the position uncertainty of the EINSTEIN X-ray source detected in the field. The star's optical position is indicated by a cross with a gap. The ellipse at the bottom left corner is the synthesized beam of $2.1 \times 1.7$ arc-second ${ }^{2}$, with position angle of $61.2^{\circ}$. 


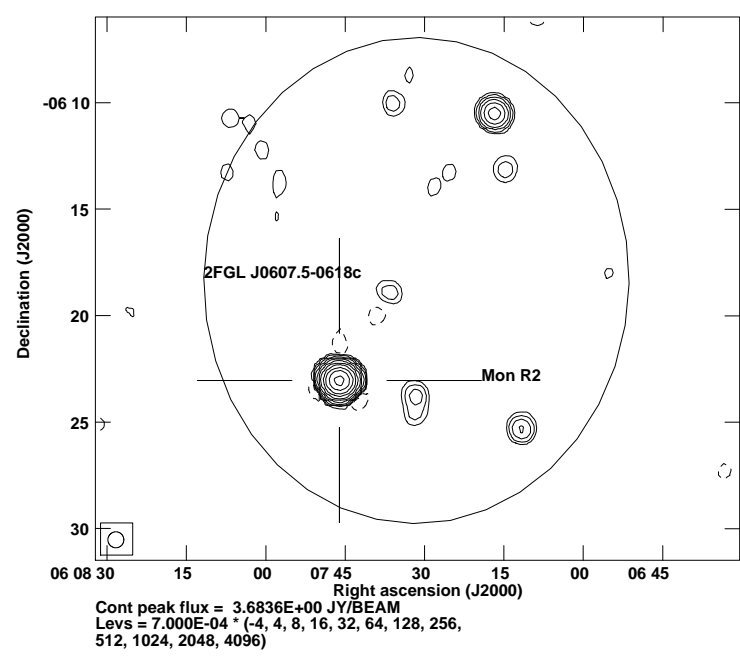

Figure 3. Contour $20 \mathrm{~cm}$ map from the NRAO VLA Sky Survey (NVSS) [14] towards the direction of the gamma-ray source 2FGL J0607.5-0618c. The error ellipse of the FermiLAT source covers most of the field. The cross with a gap marks the conspicuous radio emission of the massive star forming region Monoceros R2 that has been tentatively associated with the gamma-ray source [15]. The bottom left box illustrates the NVSS circular restoring beam of 45".

when relativistic particles are accelerated in strong shocks caused by strong magnetic activity and fast reconnection events in T-Tauri stars magnetospheres [2]. This would render the gamma-ray source 1FGL J1625.8-2429c as the closest one to the Solar System even if the high-energy photons were due to cosmic rays impacting the ambient gas. However, 1FGL J1625.8-2429c was not confirmed in the two-year release of the Fermi-LAT catalogue[10]. This could be due to either problems in the background modeling or, perhaps, to some variability effect. A detailed analysis of the whole satellite data set is still necessary in order to shed light on the reality of both the source and the proposed gamma-ray association. In any case, the lack of confirmation by Fermi LAT does not preclude the feasibility of the proposed gamma-ray emission scenario in T-Tauri stars in other systems.

\section{Conclusions}

We have reported about the current status of our search for new kinds of gamma-ray sources in the Galaxy beyond the traditional emitters such as pulsars or supernova remnants. A handful of candidate gamma-ray binaries has been identified that require future follow up observations to be confirmed or eventually ruled out. One candidate in particular, the early-type star VES 737 appears as the most interesting one. However, an EVLA observation yielded a strong upper limit so far for the system's radio emission. Finally, this paper also reviewed the situation of two nearby star forming regions tentatively associated with Fermi-LAT sources. Among them, the Monoceros $\mathrm{R} 2$ complex is the most promising place for gamma-rays produced in a YSO environment.

Acknowledgements. The authors acknowledge support of different aspects of this work by grant AYA201021782-C03-03 from the Spanish Government, and Consejería de Economía, Innovación y Ciencia of Junta de Andalucía as research group FQM-322, as well as FEDER funds. This research has made use of the SIMBAD database operated at CDS, Strasbourg, France. The National Radio Astronomy Observatory is a facility of the National Science Foundation operated under cooperative agreement by Associated Universities, Inc. The Second Palomar Observatory Sky Survey (POSS-II) was made by the California Institute of Technology with funds from the National Science Foundation, the National Geographic Society, the Sloan Foundation, the Samuel Oschin Foundation, and the Eastman Kodak Corporation.

\section{References}

[1] Mirabel, I. F., Astrophysics and Space Science, 309, 267-270 (2007)

[2] del Valle, M. V., Romero, G. E., Luque-Escamilla, P. L., Martí, J., Sánchez-Sutil, J. R., Astrophysical Journal, 738, issue 1, article Id. 115 (2011)

[3] Bosch-Ramon, V., Romero, G. E.,Araudo, A. T., Paredes, J. M., Astronomy and Astrophysics, 511, id. A8 (2010)

[4] del Valle, M. V., Romero, G. E., Astronomy and Astrophysics, 543, id. A56 (2012)

[5] Munar-Adrover, P., Paredes, J. M., Romero, G. E., Astronomy and Astrophysics, 530, id. A72 (2011)

[6] Hardorp, J., et al., http://cdsarc.u-strasbg.fr/viz-bin/Cat?III/76A (1959-1965)

[7] Wackerling, L. R., Monthly Notices of the Royal Astronomical Society, 73, 153-319 (1970)

[8] Martí, J., Paredes, J. M., Ribó, M., Astronomy and Astrophyscis, 338, L71-L74 (1998)

[9] Combi, J. A., et al., Astrophysics and Space Science, 331, 53-61 (2011)

[10] Nolan , P. L., et al., Astrophysical Journal Supplement, 199, 31 (2012)

[11] Coyne, G. V., et al., Vatican Observatory Publications, 1, 181 (1974)

[12] Paredes, J. M., Ribó, M., Martí, J., Astronomy and Astrophyscis, 394, 193-203 (2002)

[13] Haakonsen, C. B., Rutledge, R. E., Astrophysical Journal Supplement, 184, 138-151 (2009)

[14] Condon, J. J., et al., Astronomical Journal, 115, 1693-1716 (1998)

[15] Martí, J., Luque-Escamilla, P. L., Muñoz-Arjonilla, A. J., et al., Astronomy and Astrophysics, 556, id. A131 (2013) 